11

\title{
Оптические свойства легированных нанопроволок во внешних электрическом и магнитном полях
}

\author{
(С) Э.П. Синявский ${ }^{1}$, С.М. Соковнич 2 ฯ \\ ${ }^{1}$ Институт прикладной физики АН Молдовы, \\ MD 2028 Кишинёв, Молдова \\ ${ }^{2}$ Приднестровский государственный университет им. Т.Г. Шевченко, \\ MD 3300 Тирасполь, Молдова \\ ๑e-mail: s_sokovnich@rambler.ru \\ Поступила в редакцию 19.05.2020 г. \\ В окончательной редакции 19.05.2020 г. \\ Принята к публикации 07.07.2020 г.
}

Теоретически исследуется примесное поглощение света в квантовых проволоках в присутствии внешних электрического и магнитного полей, направленных параллельно друг другу и перпендикулярно оси нанопроволоки. Легированные в квантовой проволоке примеси моделируются потенциалом нулевого радиуса. Получены выражения для коэффициента поглощения света и изучены особенности примесного поглощения света для всех возможных направлений поляризации световой волны. В частности, в присутствии внешних полей становятся разрешёнными некоторые оптические переходы, которые запрещены в отсутствие полей. Исследуются частотные зависимости коэффициента поглощения света и влияние электрического и магнитного полей на величину коэффициента примесного поглощения света. Показана возможность управления оптическими характеристиками нанопроволок с помощью электрического поля.

Ключевые слова: нанопроволоки, примесное поглощение, внешние электрическое и магнитное поля.

DOI: $10.21883 /$ OS.2020.11.50181.155-20

\section{Введение}

В размерно-ограниченных системах (квантовые ямы (КЯ), квантовые проволоки (КП), нанотрубки) из-за размерного квантования возникают дополнительные каналы поглощения (люминесценции) электромагнитной волны, которые не существуют в объёмных материалах. Именно в таких квантовых системах может оказаться очень важным процесс рассеяния носителей на шероховатой поверхности [1], который иногда полностью определяет оптические свойства [2,3], явления переноса в наносистемах. В последние годы интенсивно исследуются оптические свойства полупроводниковых КП типа $\mathrm{ZnO}, \mathrm{GaN}$, $\mathrm{CdS}, \mathrm{InP}, \mathrm{GaAs}$, поскольку в таких квантовых системах наблюдаются большие подвижности носителей, и в них очень активны процессы рассеяния заряженных частиц на шероховатой поверхности [4-6].

В этих наноструктурах экспериментально исследовались процессы люминесценции на примесных состояниях: донорно-акцепторная люминесценция [6], люминесценция, связанная с переходом электрона из размерноквантованной зоны в акцепторные состояния [7]. Аналогичные оптические переходы наблюдались в нанопроволоках $\mathrm{ZnS}$ [8], InN [9]. Привлекательность исследования оптических свойств КП связана с тем, что в таких одномерных квантовых системах на дне каждой размерно-квантованной зоны проводимости (валентной зоны) возникают особенности в плотности электронных состояний. Именно это обстоятельство приводит к специфическим, по сравнению с двумерными системами, особенностям частотных зависимостей коэффициента поглощения и люминесценции электромагнитной волны. Заметим, что заметное влияние размерного квантования на оптические характеристики КП возникает при достаточно больших радиусах нанопроволок $(R \geq 1000 \AA[4])$, а описание кинетических свойств таких наносистем в модели параболического потенциала в настоящее время часто применяется и является вполне обоснованным $[10,11]$.

\section{Постановка задачи. Общие соотношения}

В настоящей работе исследуются оптические свойства легированных КП в модели параболического потенциала в присутствии поперечных электрического $\mathbf{F}$ и магнитного Н полей. Пусть магнитное поле направлено перпендикулярно оси нанопроволки $(\mathbf{H} \perp O x)$ и электрическое поле $\mathbf{F} \| \mathbf{H}$. Тогда в калибровке Ландау для векторного потенциала $\mathbf{A}(-H y, 0,0)$ гамильтониан для электрона с эффективной массой $m_{e}$ принимает вид

$$
\begin{aligned}
\hat{H}= & \frac{1}{2 m_{e}}\left[\left(\hat{p}_{x}-\frac{e}{c} H y\right)^{2}+\hat{p}_{y}^{2}+\hat{p}_{z}^{2}\right] \\
& +\frac{m_{e} \omega^{2}}{2}\left(y^{2}+z^{2}\right)+e F z .
\end{aligned}
$$

Согласно (1), потенциальная энергия носителей вдоль ocи $\mathrm{Oz}$ описывается параболой, минимум которой в 
электрическом поле находится в точке $z_{\min }=-\frac{e F}{m_{e} \omega^{2}}$. Следовательно, с ростом электрического поля положение минимума удаляется от оси симметрии нанопроволоки. Если примеси находятся на этой оси симметрии, то с ростом электрического поля электроны удаляются от локализованной примеси. В результате уменьшается перекрывание волновых функций непрерывного спектра и связанного состояния, что приводит к заметным изменениям оптических характеристик. Если же примесь расположена не на оси нанопроволоки, то присутствие электрического поля может увеличить перекрывание волновых функций. Таким образом, меняя величину электрического поля, можно управлять оптическими процессами в легированных КП.

Волновые функции $\Psi_{\alpha}^{(0)}(\mathbf{r})$ и собственные значения уравнения Шредингера с гамильтонианом (1) легко найти по аналогии с [10]. В результате собственные значения энергии определяются соотношением

$$
\begin{gathered}
E_{\alpha}=\frac{\hbar^{2} k_{x}^{2}}{2 m_{e}^{*}}+\hbar \Omega_{e}(n+1 / 2)+\hbar \omega(m+1 / 2)-\Delta, \\
m_{e}^{*}=m_{e}\left(\frac{\omega}{\Omega}\right), \quad \Omega_{e}^{2}=\omega^{2}+\omega_{c}^{2}, \\
\omega_{c}=\frac{e H}{m_{e} c}, \quad \Delta=\frac{e^{2} F^{2}}{2 m_{e} \omega^{2}} .
\end{gathered}
$$

Здесь $\hbar \omega-$ энергия размерного квантования нанопроволоки, $k_{x}$ - волновой вектор электрона, направленный вдоль оси КП, $\omega_{c}$ - циклотронная частота заряженной частицы. Как непосредственно следует из (2), энергетический спектр представляет собой набор парабол с частотой $\omega$ и гибридной частотой $\Omega_{e}$. С ростом электрического поля дно размерно-квантованной зоны проводимости опускается на величину $\Delta$ в область запрещённых значений энергий. На рис. 1 представлена энергетическая структура исследуемой легированной КП. Изображены нижайшие три размерно-квантованные зоны проводимости и энергия связанного состояния $-\tilde{E}_{S 0}$.

В дальнейшем исследуем легированные в КП примеси в модели потенциала нулевого радиуса [12]. В этой модели волновые функции $\Psi_{S}(\mathbf{r})$ связанного состояния с энергией $-E_{S}$ и волновые функции непрерывного спектра $\Psi_{\alpha}(\mathbf{r})$ имеют вид

$$
\begin{gathered}
\Psi_{S}(\mathbf{r})=C_{0} G_{S}(\mathbf{r}, 0), \\
\Psi_{\alpha}(\mathbf{r})=\Psi_{\alpha}^{(0)}(\mathbf{r})+\frac{V_{0} \tilde{\Psi}_{\alpha}^{(0)}(0)}{1-V_{0} \cdot \tilde{G}_{\alpha}(0,0)} G_{\alpha}(\mathbf{r}, 0), \\
G_{S}(\mathbf{r}, 0)=\sum_{\alpha} \frac{\Psi_{\alpha}^{(0)}(\mathbf{r}) \Psi_{\alpha}^{(0) *}(0)}{E_{S}-E_{\alpha}}, \\
G_{\alpha}(\mathbf{r}, 0)=\sum_{\alpha} \frac{\Psi_{\alpha_{1}}^{(0)}(\mathbf{r}) \Psi_{\alpha_{1}}^{(0) *}(0)}{E_{\alpha}-E_{\alpha 1}-i \varepsilon} \\
\hat{H} \Psi_{\alpha}^{(0)}(\mathbf{r})=E_{\alpha} \Psi_{\alpha}^{(0)}(\mathbf{r}),
\end{gathered}
$$

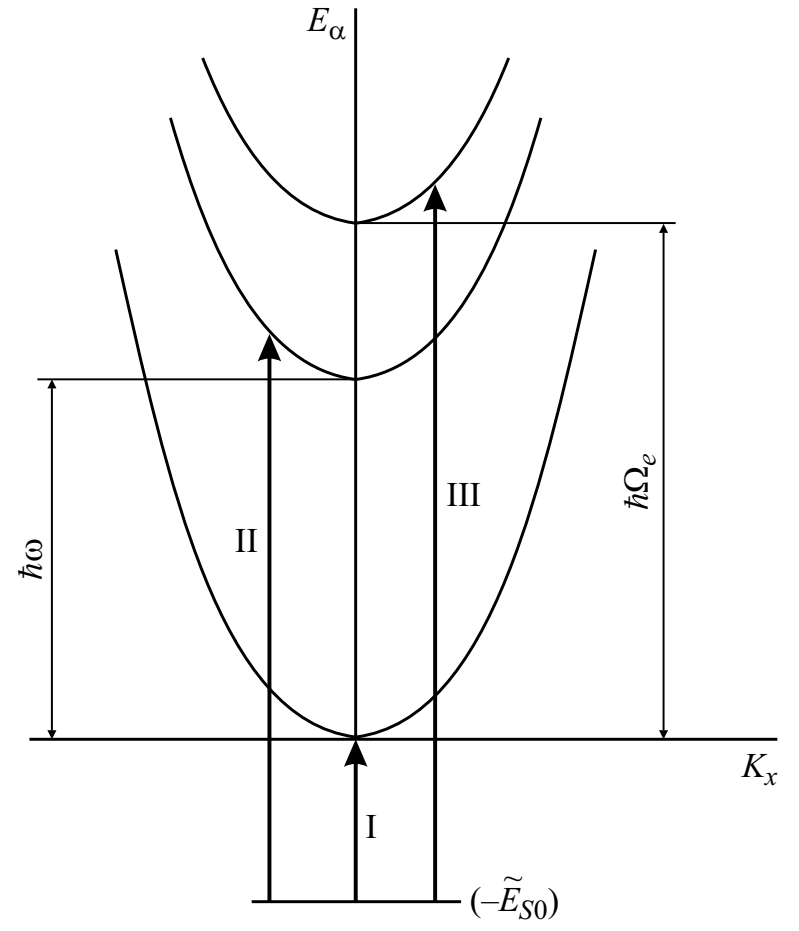

Рис. 1. Энергетическая структура исследуемой квантовой проволоки и рассматриваемые в работе оптические переходы.

$$
\tilde{A}(0)=\left.[1+\mathbf{r} \nabla] A(\mathbf{r})\right|_{\mathbf{r} \rightarrow 0} .
$$

Здесь величина $V_{0}$ определяет мощность потенциала примесного центра и связана с феноменологической постоянной $E_{S}^{0}-$ энергией связанного состояния в отсутствие полей. При записи (3) и (4) для простоты считалось, что примесь расположена в начале координат $\mathbf{r}^{\prime}(0,0,0)$, т. е. на оси симметрии размерно-квантованной проволоки, $C_{0}-$ нормировочная постоянная, $E_{S}-$ энергия связанного состояния в электрическом и магнитном полях.

Заметим, что второе слагаемое в (4) определяет вклад в волновую функцию непрерывного спектра рассеянной волны.

Для последовательного учёта особенностей в плотности электронных состояний при описании оптических переходов носителей на дно размерно-квантованной зоны в одномерных квантовых системах необходимо учитывать взаимодействие электрона с шероховатой поверхностью $[13,14]$ и (или) упругое рассеяние носителей на акустических колебаниях [2].

Коэффициент примесного поглощения света частоты $\Omega$, поляризации $\xi$ определяется соотношением

$$
\begin{gathered}
K(\Omega)=\frac{2 \pi^{2} e^{2} N_{D}}{S n_{0} c m_{e}^{2} \bar{\Omega}} \sum_{\alpha}|\langle S|\mathbf{P} \xi| \alpha\rangle|^{2} \\
\times \int d t \exp \left(\frac{i t}{\hbar}\left(E_{S}+E_{\alpha}-\hbar \Omega\right)-\Gamma_{\alpha}|t|\right), \\
\Gamma_{\alpha}=\frac{1}{\left|k_{x}\right|} \gamma,
\end{gathered}
$$




$$
\begin{gathered}
\gamma=\frac{E_{1}^{2} k_{0} T m_{e}^{2} \omega}{2 \hbar^{4} \pi \rho v^{2}}\left(1+\delta^{2}\right)^{5 / 4}+\frac{2 m_{e} \gamma_{0} \omega^{2}}{\hbar R^{2}} \\
\times\left(1+\delta^{2}\right)\left[\frac{1}{2}\left(1+\frac{1}{\sqrt{1+\delta^{2}}}\right)+\frac{2 \Delta}{\hbar \omega}\right], \\
\delta=\frac{\omega_{c}}{\omega} .
\end{gathered}
$$

Здесь $E_{1}-$ постоянная деформационного потенциала, $v$ - скорость звука в наноструктуре радиуса $R$ и плотностью $\rho, \gamma_{0}^{1 / 3}$ определяет высоту флуктуаций, $N_{D}=\frac{N}{L_{x}}$ - линейная концентрация примеси, $S-$ сечение квантовой проволоки, $n_{0}$ - показатель преломления материала КП.

\section{Обсуждение результатов}

Если примесь находится на оси КП, то функция $G_{\alpha}(\mathbf{r}, 0)$ является чётной функцией координат, и рассеянная волна в (4) не влияет на примесное поглощение света. Расчёт матричного элемента в (5) не представляет труда, и для световой волны $x$-поляризации (электромагнитная волна распространяется перпендикулярно оси КП) он определяется следующим образом:

$$
\begin{aligned}
& M_{S \alpha}^{x}=\left\langle S\left|\left(\hat{p}_{x}-\frac{e H}{c} y\right)\right| \alpha\right\rangle=C_{0} \frac{1}{\sqrt{L_{x}}}\left(\frac{\lambda_{1} \lambda_{2}}{\pi^{2}}\right)^{\frac{1}{4}} \\
& \times \exp \left(-\frac{\lambda_{1} z_{0}^{2}}{2}\right) \exp \left(-\frac{\lambda_{2} y_{01}^{2}}{2}\right) \frac{H_{m}\left(\sqrt{\lambda_{1}} z_{0}\right)}{\left(2^{n} n ! 2^{m} m !\right)^{\frac{1}{2}}} \\
& \times {\left[\frac{1}{\hbar \Omega}\left(\frac{\omega}{\Omega_{e}}\right)^{2} \hbar k_{x} H_{n}\left(-\sqrt{\lambda_{2}} y_{01}\right)-\sqrt{\frac{\hbar m_{e}}{\Omega_{e}}}\right.} \\
& \times\left.\left(\frac{n H_{n-1}\left(-\sqrt{\lambda_{2}} y_{01}\right)}{\hbar \Omega-\hbar \Omega_{e}}+\frac{1}{2} \frac{H_{n+1}\left(-\sqrt{\lambda_{2}} y_{01}\right)}{\hbar \Omega+\hbar \Omega_{e}}\right)\right] . \\
& \lambda_{1}=\frac{m_{e} \omega}{\hbar}, \quad \lambda_{2}=\frac{m_{e} \Omega_{e}}{\hbar} \\
& z_{0}=\frac{e F}{m_{e} \omega^{2}}, \quad y_{01}=\frac{\hbar \omega_{c} k_{x}}{m_{e} \Omega_{e}^{2}} .
\end{aligned}
$$

Здесь $H_{n}(z)$ - полиномы Эрмита-Чебышева.

При записи (7) использовался закон сохранения энергии: $\hbar \Omega=\tilde{E}_{S 0}+E_{\alpha}$. В отсутствие внешних полей $(H=F=0)$, учитывая (7), коэффициент примесного поглощения света $x$-поляризации может быть записан следующим образом:

$$
\begin{aligned}
K^{(x)}(\Omega) & =K_{0}\left(\frac{\hbar \omega_{f}}{\hbar \omega}\right)^{\frac{1}{2}}\left(\frac{\omega}{\Omega}\right)^{3} \\
& \times \frac{1}{\pi} \sum_{n, m}\left[\frac{H_{n}^{2}(0)}{2^{n} n !}\right]\left[\frac{H_{m}^{2}(0)}{2^{m} m !}\right] I\left(\Delta_{n m}\right), \\
K_{0} & =\frac{4 C_{0}^{2} e^{2} N_{D}}{S n_{0} c \hbar^{4} \omega^{2}}\left(2 m_{e} \hbar \omega\right)^{1 / 2},
\end{aligned}
$$

$$
\begin{gathered}
I(\tau)=\int_{0}^{\infty} \frac{x d x}{1+x(x-\tau)^{2}}, \\
\Delta_{n m}=\frac{\hbar \Omega-\hbar \omega(n+m)-\tilde{E}_{S_{0}}}{\hbar \omega_{f}} \\
\hbar \omega_{f}=\left(\frac{\hbar \gamma^{2}}{2 m_{e}}\right)^{1 / 3}, \quad H_{2 n+1}(0)=0, \\
H_{2 n}(0)=(-1)^{n} 2^{n}(2 n-1) ! !
\end{gathered}
$$

Из соотношения (8) непосредственно следует, что в отсутствие внешних полей возможны оптические переходы носителей только между примесными состояниями и чётными размерно-квантованными уровнями зоны проводимости (переход I на рис. 1 разрешен, а оптические переходы II, III запрещены). Заметим, что если при поглощении света электрон с примесного состояния переходит на дно размерно-квантованной зоны, то $\Delta_{n m}=0$ $\left(\hbar \Omega=\tilde{E}_{S 0}+\hbar \omega(n+m)\right)$ и $I(0)=\frac{2 \pi}{3 \sqrt{3}} . \tilde{E}_{S 0}=E_{S}+\hbar \omega-$ энергия связанного состояния, отсчитанная от дна зоны проводимости. Такие оптические переходы отсутствуют, если пренебречь процессами рассеяния электронов, так как при $\gamma \rightarrow 0$ получаем $I(\tau)=\pi \sqrt{\tau}$. Следовательно, процессы рассеяния носителей существенно влияют на частотную зависимость коэффициента поглощения электромагнитной волны в длинноволновой области спектра.

На рис. 2 представлена частотная зависимость коэффициента поглощения света при оптическом переходе электрона из примесного состояния $\left(\tilde{E}_{S 0}=4 \hbar \omega\right)$ на уровень $n=m=0$ - пик 1 (оптический переход I на

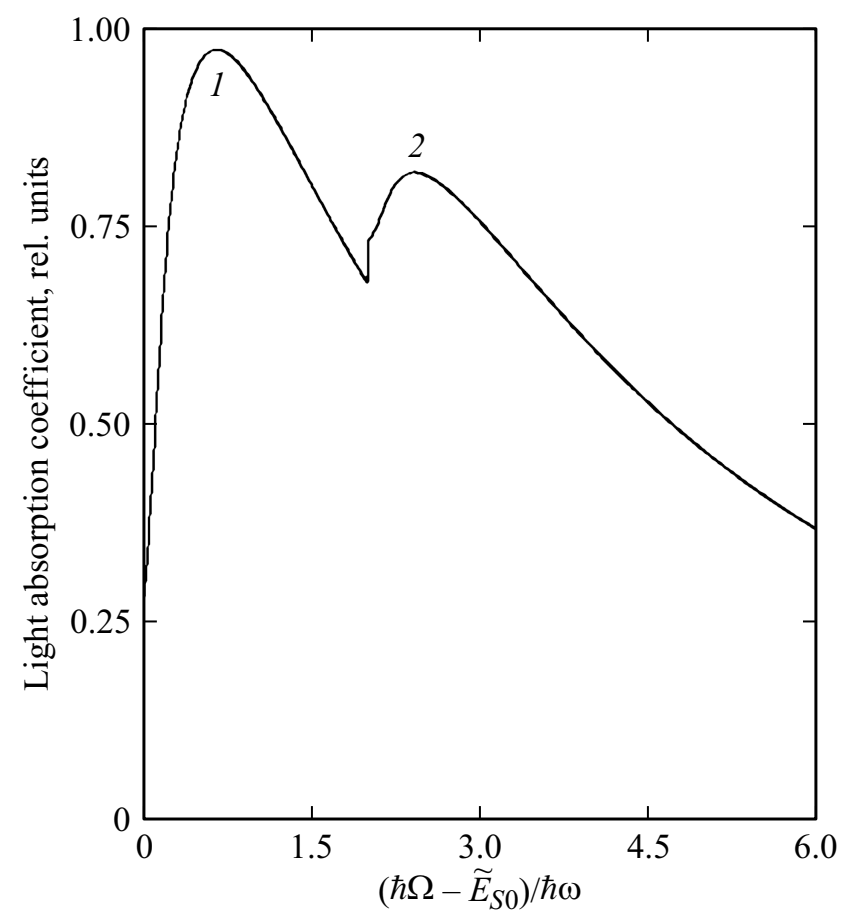

Рис. 2. Частотная зависимость коэффициента поглощения света в отсутствии внешних полей $\left(\tilde{E}_{S 0}=4 \hbar \omega\right)$. 
рис. 1) и на уровни $n=0, m=2$ и $n=2, m=0$ (пик 2). Расчёты проводились при $\hbar \omega_{f}=0.1 \hbar \omega$.

В присутствии поперечных электрического и магнитного полей коэффициент примесного поглощения света (переход I на рис. 1) с учётом (7) и (8) определяется следующим выражением (для простоты пренебрегаем процессами рассеяния носителей $\left(\gamma=0\right.$, поэтому $\left.\left.\Delta_{0}>0\right)\right)$ :

$$
\begin{gathered}
K_{S, 00}^{(x)}\left(\Delta_{0}\right)=K_{0} \frac{1}{\left(1+\delta^{2}\right)^{\frac{1}{4}}}\left(\frac{\omega}{\Omega}\right)^{3} \sqrt{\frac{\Delta_{0}}{\hbar \omega}} \exp \left(-\frac{2 \Delta}{\hbar \omega}\right) \\
\times \exp \left[-\frac{2 \delta^{2}}{\left(1+\delta^{2}\right)^{1 / 2}} \frac{\Delta_{0}}{\hbar \omega}\right]\left(1+\frac{\Omega}{\Omega+\Omega_{e}} \delta^{2}\right)^{2}, \\
\Delta_{0}=\hbar \Omega-\tilde{E}_{0} \geq 0, \\
\tilde{E}_{0}=E_{S}+\frac{1}{2} \hbar\left(\Omega_{e}+\omega\right)-\Delta .
\end{gathered}
$$

С ростом напряжённости электрического поля коэффициент поглощения света уменьшается (наличие множителя $\exp \left(-\frac{2 \Delta}{\hbar \omega}\right)$ в (9)), что связано с ослаблением перекрывания волновых функций связанного состояния и непрерывного спектра. На рис. 3 представлена частотная зависимость коэффициента поглощения света в относительных единицах (переход I на рис. 1) в присутствии поперечного электрического поля и в отсутствие магнитного поля. Кривые 1, 2, 3 получены при $\frac{\Delta}{\hbar \omega}=0$, 0.2 и 0.5 соответственно (расчёты проводились при $\left.\tilde{E}_{S 0}=4 \hbar \omega, \delta=0\right)$.

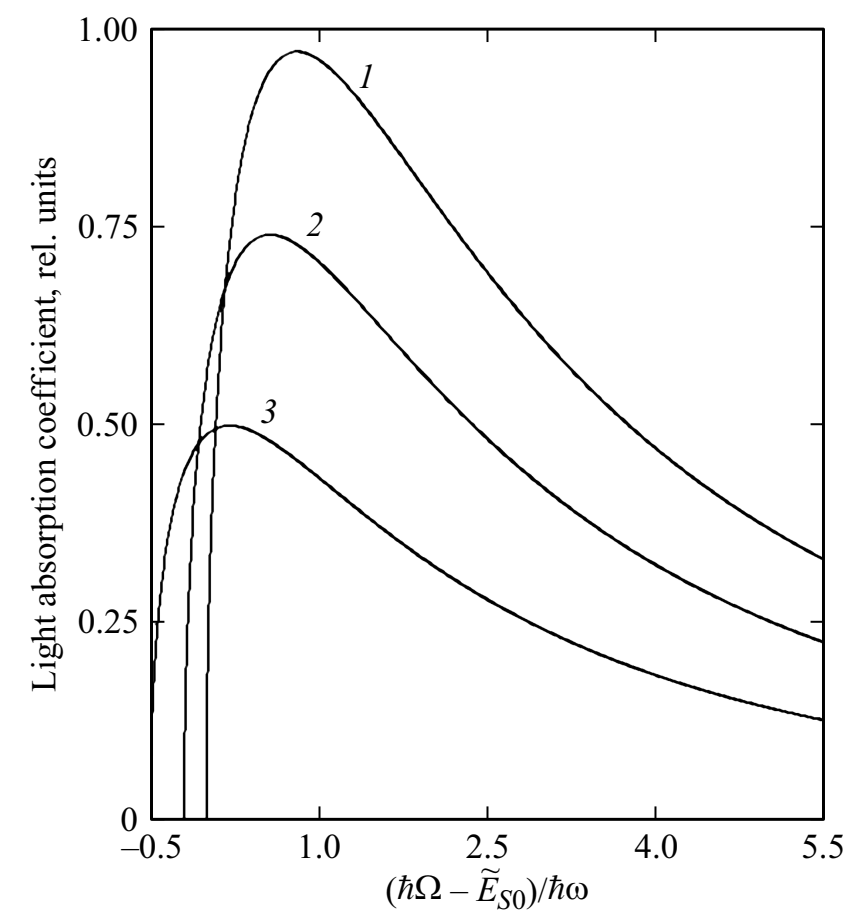

Рис. 3. Частотная зависимость коэффициента поглощения света в относительных единицах для различных значений напряжённости электрического поля.
С ростом напряжённости электрического поля дно размерно-квантованной зоны сдвигается в область запрещённых значений энергии на величину $\Delta$, поэтому экстремумы коэффициента поглощения электромагнитной волны смещаются в длинноволновую область спектра. Как показали исследования, с ростом напряжённости магнитного поля увеличивается энергия связи примесного состояния $\tilde{E}_{0}$, поэтому максимум коэффициента примесного поглощения света смещается в высокочастотную область спектра.

Рассмотрим оптические переходы в КП из связанного состояния на возбуждённые состояния размерноквантованной зоны проводимости. Коэффициент примесного поглощения $x$-поляризованного света на первое размерно-квантованное состояние (оптический переход II на рис. 1), согласно (5) и (7), с $m=1, n=0$ определяется соотношением

$$
\begin{gathered}
K_{S, 10}^{(x)}\left(\Delta_{1}\right)=4\left(\frac{\Delta}{\hbar \omega}\right) K_{S, 00}^{(x)}\left(\Delta_{1}\right), \\
\Delta_{1}=\hbar \Omega-\tilde{E}_{0}-\hbar \omega>0 .
\end{gathered}
$$

При записи (10) пренебрегалось взаимодействием носителей с фононами и рассеянием на шероховатой поверхности, поэтому $\Delta_{1}>0$. Как непосредственно следует из (10), поперечное электрическое поле разрешает оптические переходы II (рис. 1), при этом частотная зависимость коэффициента поглощения света (10) качественно не отличается от случая перехода на нижайшее размерно-квантованное состояние. Заметим, что в магнитном поле первый размерно-квантованный уровень расщепляется, что позволяет исследовать оптические переходы электрона из связанного состояния на гибридные состояния (например, оптический переход III на рис. 1). В этом случае коэффициент поглощения $x$-поляризованной волны принимает вид (процессами рассеяния зонных электронов пренебрегаем)

$$
\begin{aligned}
& K_{S, 01}^{(x)}\left(\Delta_{2}\right)=K_{0} \delta^{2}\left(1+\delta^{2}\right)^{\frac{3}{4}}\left(\frac{\omega}{\Omega}\right)^{3} \sqrt{\frac{\hbar \omega}{\Delta_{2}}} \\
& \times \exp \left(-\frac{2 \Delta}{\hbar \omega}-\frac{2 \Delta_{2}}{\hbar \omega} \frac{\delta^{2}}{\left(1+\delta^{2}\right)^{\frac{1}{4}}}\right) \\
& \times\left[\frac{2 \Delta_{2}}{\hbar \omega_{e}}\left(1+\frac{\hbar \omega}{\hbar \omega+\hbar \Omega_{e}} \delta^{2}\right)+\frac{\hbar \Omega \hbar \Omega_{e}}{(\hbar \Omega)^{2}-\left(\hbar \Omega_{e}\right)^{2}}\right]^{2}, \\
& \Delta_{2}=\hbar \Omega-\tilde{E}_{0}-\hbar \Omega_{e}>0 .
\end{aligned}
$$

Согласно (11), зависимость коэффициента поглощения света от напряжённости поперечного электрического поля такая же, как для примесного поглощения света при оптическом переходе электрона на нижайшее размерно-квантованное состояние зоны проводимости. C ростом напряжённости магнитного поля максимум поглощения электромагнитной волны сдвигается в область высоких частот, так как увеличиваются энергия 
связи локализованного состояния и энергия гибридного состояния $\hbar \Omega_{e}$.

Исследуем примесное поглощение света $y$-поляризации (электромагнитная волна распространяется перпендикулярно оси КП, а вектор поляризации перпендикулярен $\mathbf{F}$ и $\mathbf{H})$.

Коэффициент поглощения света, связанный с оптическими переходами носителя из примесного состояния на основное размерно-квантованное состояние $(m=n=0)$, записывается в виде

$$
\begin{gathered}
K_{s, 00}^{(y)}\left(\Delta_{0}\right)=K_{0}\left(1+\delta^{2}\right)^{3 / 4}\left(\frac{\omega}{\Omega}\right) \frac{\delta^{2}}{\left(\hbar \Omega_{e}+\hbar \Omega\right)^{2}} \\
\times \exp \left(-\frac{2 \Delta}{\hbar \omega}\right) \exp \left(-z_{0}\right)\left(\frac{\Delta_{0}}{\hbar \omega}\right)^{1 / 2}, \\
z_{0}=\frac{\Delta_{0}}{\hbar \omega} \frac{2 \delta^{2}}{\sqrt{1+\delta^{2}}} .
\end{gathered}
$$

При записи (12) пренебрегалось рассеянием носителей на фононах и на шероховатой поверхности, поэтому $\Delta_{0}>0$. Как непосредственно следует из (12), магнитное поле стимулирует поглощение света $y$-поляризации, при этом с ростом напряжённости поперечного электрического поля поглощение электромагнитной волны уменьшается и максимум поглощения смещается в длинноволновую область спектра. При увеличении напряжённости однородного магнитного поля коэффициент поглощения растёт, но при больших магнитных полях $(\delta>1)$ уменьшается в высокочастотной области спектра. Заметим, что с ростом $\delta$ максимум поглощения смещается в область высоких частот из-за увеличения гибридной частоты $\Omega_{e}$. При оптическом переходе электрона на возбуждённое размерно-квантованное состояние $(m=1$, $n=0)$ коэффициент поглощения принимает вид

$$
\begin{aligned}
K_{s, 10}^{(y)}\left(\Delta_{10}\right) & =4 \frac{\Delta}{\hbar \omega} K_{s, 00}^{(y)}\left(\Delta_{10}\right), \\
\Delta_{10} & =\Delta_{0}-\hbar \omega .
\end{aligned}
$$

Следовательно, такое поглощение света возможно только в присутствии поперечных электрического и магнитного полей. При этом с ростом напряжённости электрического поля коэффициент поглощения сначала растёт (максимум достигается при $\frac{2 \Delta}{\hbar \omega}=1$ ), а затем экспоненциально убывает (уменьшается перекрытие волновых функций в связанном состоянии и в размерноквантованной зоне проводимости).

Рассмотрим особенности примесного поглощения $z$ поляризованной электромагнитной волны (вектор поляризации поглощаемого света параллелен напряжённостям внешних полей). Коэффициент поглощения света без учёта процессов рассеяния зонных электронов запи- сывается следующим образом:

$$
\begin{aligned}
& K^{(z)}(\Omega)=\frac{1}{2} K_{0}\left(1+\delta^{2}\right)^{\frac{3}{4}} \exp \left(-\frac{2 \Delta}{\hbar \omega}\right) \\
& \times \sum_{m n}\left(\frac{\hbar \omega}{\Delta_{m n}^{(0)}}\right)^{\frac{1}{2}}\left(\frac{\omega}{\Omega}\right) \exp \left(-z_{m n}\right) \frac{H_{n}^{2}\left(\sqrt{z_{m n}}\right)}{2^{m} 2^{n} m ! n !} \\
& \times\left(\frac{m H_{m-1}\left(\sqrt{\frac{2 \Delta}{\hbar \omega}}\right)}{\hbar \Omega-\hbar \omega}-\frac{H_{m+1}\left(\sqrt{\frac{2 \Delta}{\hbar \omega}}\right)}{2(\hbar \omega+\hbar \Omega)}\right)^{2}(\hbar \omega)^{2}, \\
& z_{m n}=\frac{\Delta_{m n}^{(0)}}{\hbar \omega} \frac{2 \delta^{2}}{\sqrt{1+\delta^{2}}}, \\
& \Delta_{m n}^{(0)}=\Delta_{0}-\hbar \omega m-\hbar \Omega_{e} n .
\end{aligned}
$$

Как непосредственно следует из (14), в отсутствие внешних полей $(F=H=0)$ примесное поглощение света возможно на состояния размерно-квантованной зоны проводимости, когда $m-$ нечётное, а $n-$ чётное. Следовательно, как и в случае $y$-поляризованного света, оптические переходы электрона из связанного состояния в нижайшее состояние зоны проводимости в отсутствие внешних полей запрещены.

Коэффициент поглощения $z$-поляризованной электромагнитной волны при переходе электрона из локализованного состояния в основное состояние размерноквантованной зоны проводимости $(m=n=0)$ во внешних полях определяется соотношением $\left(\Delta_{0}>0\right)$

$$
\begin{gathered}
K_{s, 00}^{(z)}(\Omega)=\frac{1}{2} K_{0} \exp \left(-\frac{2 \Delta}{\hbar \omega}-z_{0}\right)\left(1+\delta^{2}\right)^{3 / 4} \\
\times\left(\frac{\hbar \omega}{\Delta_{0}}\right)^{1 / 2}\left(\frac{\omega}{\Omega}\right)\left(\frac{2 \Delta}{\hbar \omega}\right) \frac{(\hbar \omega)^{2}}{(\hbar \omega+\hbar \Omega)^{2}} .
\end{gathered}
$$

Следовательно, поперечное электрическое поле разрешает такие оптические переходы, и с ростом напряжённости электрического поля поглощение света увеличивается (при $\frac{2 \Delta}{\hbar \omega}=1$ достигает максимума), но при больших полях $\left(\frac{2 \Delta}{\hbar \omega}>1\right)$ оно уменьшается, что связано с уменьшением перекрывания волновых функций связанного состояния и непрерывного спектра. С ростом $F$ максимум поглощения сдвигается в длинноволновую область, так как уменьшается энергия связанного состояния $\tilde{E}_{0}$.

Выражение для коэффициента примесного поглощения света, связанное с переходом электрона на первое размерно-квантованное состояние $(n=0, m=1)$, 
нетрудно получить из $(14)\left(\Delta_{10}^{(0)}>0\right)$ :

$$
\begin{aligned}
& K_{s, 10}^{(z)}(\Omega)=\frac{1}{2} K_{0} \exp \left(-\frac{2 \Delta}{\hbar \omega}\right) \exp \left(z_{10}\right)\left(1+\delta^{2}\right)^{\left(\frac{3}{4}\right)} \\
& \times\left(\frac{\hbar \omega}{\Delta_{10}^{(0)}}\right)^{\frac{1}{2}}\left(\frac{\omega}{\Omega}\right)\left(\frac{1}{\hbar \Omega-\hbar \omega}-\frac{\frac{4 \Delta}{\hbar \omega}-1}{\hbar \omega+\hbar \Omega}\right)^{2}(\hbar \omega)^{2} .
\end{aligned}
$$

Следовательно, поглощение электромагнитной волны возможно и в отсутствии внешних электрического и магнитного полей. С ростом напряжённости электрического поля подавляется такое поглощение света, а максимум сдвигается в длинноволновую область спектра.

Коэффициент примесного поглощения электромагнитной волны $z$-поляризации, связанный с переходом электрона на первое гибридное состояние (переход III на рис. 1$)$, определяется соотношением $\left(\Delta_{01}^{(0)}>0\right)$

$$
\begin{aligned}
& K_{s, 01}^{(z)}(\Omega)=32 K_{0} \frac{\exp \left(-\frac{2 \Delta}{\hbar \omega}\right)}{\hbar \omega} \exp \left(-z_{01}^{2}\right) \\
& \times\left(1+\delta^{2}\right)^{-1 / 4} \delta^{2}\left(\frac{\Delta_{01}^{(0)}}{\hbar \omega}\right)^{1 / 2}\left(\frac{\omega}{\Omega}\right) \frac{\Delta \hbar \omega}{(\hbar \omega+\hbar \Omega)^{2}} .
\end{aligned}
$$

Рассматриваемое поглощение света возможно только в электрическом и магнитном полях. При этом с ростом напряжённости однородного магнитного поля поглощение сначала увеличивается, а в области высоких частот при $\delta>1$ уменьшается. Последнее обстоятельство связано с уменьшением перекрывания волновых функций связанного состояния и непрерывного спектра в магнитном поле.

\section{Выводы}

Проведены исследования по влиянию электрического и магнитного полей на примесное поглощение света в КП. Если примесь легирована на расстоянии $z_{c}$ от оси симметрии, то влияние поперечного электрического поля на коэффициент поглощения электромагнитной волны в основном определяется множителем $\exp \left(-2\left[\left(\frac{2 \Delta}{\hbar \omega}\right)^{1 / 2}+\frac{z_{c}}{R}\right]^{2}\right)$. Следовательно, при $z_{c}<0$ с ростом $\Delta$ (т.е. с увеличением напряжённости электрического поля) коэффициент примесного поглощения сначала увеличивается, а при $\left(\frac{2 \Delta}{\hbar \omega}\right)^{1 / 2}>\frac{\left|z_{c}\right|}{R}$ экспоненциально уменьшается. Такое немонотонное изменение поглощения света позволяет определить положение $\delta$ легированной примеси. Внешние электрическое и магнитное поля значительно увеличивают каналы примесного поглощения электромагнитной волны, что безусловно расширяет возможности применения легированных КП в оптоэлектронике.

\section{Конфликт интересов}

Авторы заявляют, что у них нет конфликта интересов.

\section{Список литературы}

[1] Sakaki H., Noda T., Hurakawa K. et.al. // Appl. Phys. Lett. 1987. V. 51. N 23. P. 1934. doi $10.1063 / 1.98305$

[2] Синявский Э.П., Костюкевич Н.С. // Опт. и спектр. 2013. T. 114. № 2. C. 225; Sinyavskii E.P., Kostyukevich N.S. // Opt. Spectrosc. 2013. V. 114. N 2. P. 205. doi 10.1134/S0030400X13020276

[3] Unuma T., Takahashi T., Noda T. et.al. // J. Appl. Phys. 2001. V. 78. N 22. P. 3448. doi $10.1063 / 1.1376154$

[4] Agarwal R., Lieber C.M. // Appl. Phys. A. 2006. V. 85. N 3. P. 209. doi 10.1007/s00339-006-3720-z

[5] Iahn V., L'ahnemann J., Pfuller C. et.al. // Phys. Rev. B. 2012. V. 85. 045323. doi 10.1103/PhysRevB.85.045323

[6] Xiao-Yan Yan, Cheng-Bao Yao, Jin Li et.al. // Optical Materials. 2016. V. 55. P. 73. doi 10.1016/j.optmat.2016.03.016

[7] Xianghui Zhang, Ye Zhang, Yipu Song, Zhe Wang, Dapeng Yu. // Phys. E. Low-dimensional Syst. Nanostructures. 2005. V. 28. N 1. P. 1. doi 10.1016/j.physe.2004.12.022

[8] Sowmya Kolli, Chandra Shekhar Pendyala et.al. // J. Lumin. 2013. V. 141. P. 162. doi 10.1016/j.jlumin.2013.03.041

[9] Becnakker C.W.J., van Houten H. Solid States Physics Semiconductor Heterostructures and Nanosructures. / Ed. by Ehrenreich H. N.Y.: Academic Press, 1991. 128 p.

[10] Гейлер В.А., Маргулис В.А. // ЖЭТФ. 1998. Т. 113. № 4. C. 1376.

[11] Гейлер В.А., Маргулис В.А. // ФТП. 1999. Т. 33. № 9. C. 1141.

[12] Демков Ю.Н., Островский В.Н. Метод потенциалов нулевого радиуса в атомной физике. Л.: Изд-во ЛГУ, 1975. $240 \mathrm{c}$.

[13] Синявский Э.П., Карапетян С.А. // ФТП. 2012. Т. 46. № 8. C. 1032.

[14] Синявский Э.П., Хамидуллин Р.А. // ФТП. 2002. Т. 36. No 8. C. 989. 